\title{
Review of: "Human upper-airway respiratory airflow: In vivo comparison of computational fluid dynamics simulations and hyperpolarized 129Xe phase contrast MRI velocimetry"
}

\author{
Jianan Zhao ${ }^{1}$ \\ 1 Oklahoma State University
}

Potential competing interests: The author(s) declared that no potential competing interests exist.

In this study, the authors proposed an innovative method for validating the CFD prediction of the velocity field in the human respiratory system by comparing with in vivo data. Specifically, the phase contrast (PC) velocimetry magnetic resonance imaging (MRI) of inhaled hyperpolarized ${ }^{129}$ Xe gas was used as a noninvasive reference to which airflow velocities calculated via CFD can be compared. The structure of this paper was well organized and the logic was clear and sound. The results and discussion are very detailed and thorough. This is a very promising method for the respiratory CFD model validation, although limitations and shortcomings need to be improved in the future studies.

Below are my thoughts and comments:

1. Maybe the authors can add the information about the integral length scale of the final mesh so that readers can better understand how much turbulence kinetic energy was resolved using the LES method. Also, in terms of the temporal resolution, the authors said "the CFD velocity results did not change below $0.05 \mathrm{~s}$ ". This is a very vague statement and a time step of $0.05 \mathrm{~s}$ seems pretty large compared with other respiratory CFD papers using LES method (please refer to doi.org/10.1016/j.ejps.2019.03.025). What are the convergence criteria for LES simulation?

2. Is the inlet boundary condition exactly same as the flow profile measured by experiment, which is shown in Fig. 1? The reason why I ask is because there are some disagreements in the velocity field between the CFD and PC MRI results. Also, does the glottis motion absent from the CFD simulation affect the comparison results?

3. In page 7 , it says “ $\ldots$ the results of the LES produced the best correlation (URANS: $r=0.65$, LES: $r=$ $0.68) "$. Is there a huge difference in the results between $r=0.65$, LES: $r=0.68$ ? To me, the difference in $r$ value between the URANS and LES results is not obvious. So, maybe URANS is able to generate results close enough to LES results while taking less computational time?

4. What is the total physical time simulated using CFD?

5. The advantage of LES method is that it can resolve the majority of turbulence while model the small scale eddies. Did the authors compare any turbulence related properties such as turbulence kinetic 
energy or turbulence intensity between CFD and PC MRI results?

6. In page 10 last paragraph, what is the "general lack of directional airflow"? Does it mean there is little airflow motion in the RL direction at current Re (mostly laminar flow)? Have the author considered to perform a comparison at a higher flow rate so the secondary flow is obvious enough to be observed? 